\title{
Liquid Jet Instability Under Gravity Effects
}

\author{
Ghobad Amini ${ }^{1}$ and Matthias Ihme ${ }^{2}$ \\ Department of Aerospace Engineering, University of Michigan, Ann Arbor, MI 48109
}

\begin{abstract}
The effect of gravity on the onset and growth rate of absolute and convective instabilities in viscous liquid jets is studied. To this end, a spatial linear stability analysis of the Cosserat's equations is performed. A multiscale expansion technique is employed and dispersion relation and expressions for the perturbation amplitude are derived to evaluate the growth rate of axisymmetric disturbances. Model-results for zero gravity are compared with classical results of hydrodynamic instability, confirming the validity of this approach. It is shown that gravity increases both the instability growth rate and the cutoff frequency below which disturbances are spatially amplified and above which they are damped. The critical Weber number, demarcating the transition between convective and absolute instability is determined as function of Reynolds and Froude numbers. It is found that, by increasing the acceleration of gravity, the critical Weber number decreases. This result explains the reason for shifting the transition between dripping and jetting to lower Weber numbers in the case of increasing gravity.
\end{abstract}

\section{Introduction}

Iinjec Nons NSTABILITY and breakup of liquid jets is important for a wide range of applications, including liquid fuel injection, coating, drug delivery, food preparation, and ink-jet printing [1]. By neglecting surrounding-gas effects and gravity, Rayleigh [2] demonstrated that the origin of the jet breakup is the hydrodynamic instability. He showed that a circular cylindrical liquid jet is unstable with respect to disturbances of wavelengths larger than the jet circumference. Keller et al. [3] argued that unstable disturbances grow over space as they are convected along the downstream direction. Leib \& Goldstein $[4,5]$ studied the absolute instability of liquid jets, which arises from a saddle-point singularity in the dispersion relation. In contrast to the convective instability analysis of Keller et al [3], the unstable disturbances in an absolutely unstable jet propagate in both upstream and downstream directions.

Although gravitational effects were studied frequently in some processes such as fiber formation [1], the effect of gravity on the capillary instability of low-speed liquid jets is rarely studied. The reason for this is the complexity of breakup processes even without considering gravity.

In the Rayleigh breakup regime, which occurs at low-speed flows, the surrounding gas effects are negligible and the breakup mechanism is due to capillary pinching. In this regime, at lower speeds, gravity is a controlling instability mechanism and cannot be ignored. Therefore, providing a physical understanding about gravitational effects on the primary jet break-up has the potential to better control breakup length and drop-size, which is vital in numerous practical applications such as microencapsulation, DNA-biochips deposition, or bottle filling process [1, $6,7]$.

The three-dimensional equations describing the nonlinear motion of gravity-affected free-surface flows will be complicated if analytical solutions are to be obtained. Therefore generating simplified equations that capture the essential nonlinear physics of the problem is very useful. Green [8] presented a nonlinear one-dimensional model for a straight, circular, viscous jet using the basic theory of the one-dimensional Cosserat's continuum equations. Cosserat's equations can be systematically derived from the Navier-Stokes equations through a Taylor-series expansion [9] or through an averaging method over the cross-sectional plane [10]. In the second method, using a Galerkin averaging method and integrating the Navier-Stokes equations over the jet's cross-section, a system of onedimensional equations for arbitrarily high order can be obtained [10]. Boundary conditions include the attachment of the material to the surface (kinematic boundary condition) and the discontinuity in the normal traction balance, due to surface tension (kinetic boundary condition). Asymptotically, the leading-order versions of these integrodifferential equations for a slender jet result in the Cosserat's equations. The resulting governing equations are highorder nonlinear partial differential equations [1]. This 1-D model, inherently containing radial inertia effects, describes the jet profile, velocity and pressure as function of the axial coordinate only, and simplifies the analysis

\footnotetext{
${ }^{1}$ Postdoctoral Scholar, AIAA Member.

${ }^{2}$ Assistant Professor, AIAA Member.
} 
considerably. Bogy [11-14] used the Cosserat's equations successfully to study the stability of circular liquid jets in linear and nonlinear regimes. Most recently, Amini \& Dolatabadi $[15,16]$ analyzed the temporal and spatial stability of viscous elliptic jets using the Cosserat's theory in the absence of gravitational forces.

The objective of this work is to investigate the Rayleigh instability of viscous liquid jets under conditions corresponding to positive Froude numbers. To this end, a multiscale expansion of the one-dimensional Cosserat's equations is performed. The mean flow is obtained from the numerical solution of the steady-state Cosserat's equations. The governing equations and linearized form are presented in the next section. From the linearized Cosserat's equations, an analytic dispersion relation is derived. Using this relation, the instability behavior is parametrically investigated in Sec. III. The paper finishes with conclusion.

\section{Governing Equations}

The present analysis considers the instability of a liquid jet in the presence of viscosity, surface tension, and gravitational forces. The complete set of Cosserat's equations includes conservation equations for mass and momentum, which after eliminating pressure among axial and radial momentum equations [17], are written in non-dimensional form as

$$
\begin{gathered}
\left(r^{2}\right)_{t}+\left(r^{2} v\right)_{z}=0 \\
r^{2}\left(v_{t}+v v_{z}\right)-\frac{1}{2} r^{3} r_{z}\left(v_{z t}+v v_{z z}-\frac{1}{2} v_{z}^{2}\right)-\frac{1}{8} r^{4}\left(v_{z z t}+v v_{z z z}\right)= \\
\frac{1}{\mathrm{We}}\left(\frac{r}{\left(1+r_{z}^{2}\right)^{1 / 2}}+\frac{r^{2} r_{z z}}{\left(1+r_{z}^{2}\right)^{3 / 2}}\right)_{z}+\frac{1}{\operatorname{Re}}\left(3 r^{2} v_{z}-\frac{1}{8}\left(r^{4} v_{z z}\right)_{z}\right)_{z}+\frac{r^{2}}{\mathrm{Fr}}
\end{gathered}
$$

where $r$ is the liquid jet-radius and $v$ is the velocity component in axial direction (See Fig.1). These variables are functions of axial distance, $z$, and time, $t$. Subscripts $z$ and $t$ represent partial derivative with respect to axial distance and time, respectively. Eq. (2) describes the balance between momentum, on the left, and capillary, viscosity, and gravitational forces, on the right. All quantities in Eq. (2) are non-dimensionalized by using the initial jet radius $R^{*}$ and axial velocity $V^{*}$ as

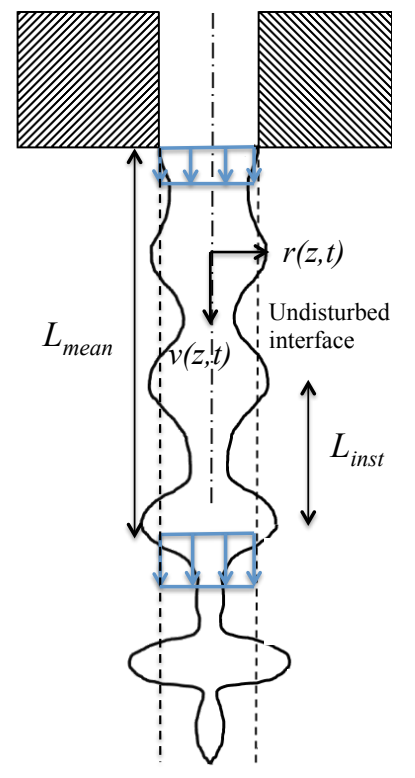

Fig. 1. Schematic of liquid jet and relevant quantities.

$$
r=\frac{r^{*}}{R^{*}}, \quad v=\frac{v^{*}}{V^{*}}, \quad z=\frac{z^{*}}{R^{*}}, \quad t=\frac{t^{*} V^{*}}{R^{*}},
$$

where an asterisk refers to a dimensional quantity. Through this non-dimensionalization, the following dimensionless groups can be identified:

Reynolds number:

$$
\operatorname{Re}=\frac{\rho^{*} R^{*} V^{*}}{\mu^{*}}
$$

Froude number:

$$
\operatorname{Fr}=\frac{V^{* 2}}{g^{*} R^{*}}
$$

Weber number:

$$
\mathrm{We}=\frac{\rho^{*} R^{*} V^{* 2}}{\sigma^{*}}
$$

where $\mu^{*}$ is the dynamic viscosity, $\rho^{*}$ is the density, $\sigma^{*}$ is the surface tension, and $g^{*}$ is the gravitational acceleration. Another dimensionless number commonly used is the Bond number, defined as,

Bond number:

$$
\mathrm{Bo}=\frac{\rho^{*} R^{*^{2}} g^{*}}{\sigma^{*}}=\frac{W e}{F r} .
$$




\section{Linear Stability Analysis}

\section{A. Solution of base flow}

The stability analysis presented in the next section, is performed around a steady-state base flow. To perform a local linear stability analysis, all state-variables in Eqs. (1) and (2) are decomposed into a mean and a fluctuating quantity, viz.,

$$
r=\bar{r}+r^{\prime} \quad \text { and } \quad v=\bar{v}+v^{\prime}
$$

In the absence of gravity, an axis-parallel mean flow with a constant jet radius, i.e. $\bar{r}=1$, is a solution of the unperturbed equation. However due to gravity, the liquid jet may contract in a downward-pointing motion. Therefore, the streamwise evolution of the base flow must be considered. In the following analysis, the base flow is obtained from the numerical solution of the steady-state form of Cosserat's equations (1) and (2) as

$$
\begin{gathered}
\left(\bar{r}^{2} \bar{v}\right)_{z}=0 \\
\bar{r}^{2} \overline{v v}_{z}-\frac{1}{2} \bar{r}^{3} \bar{r}_{z}\left(\bar{v}_{z z}-\frac{1}{2} \bar{v}_{z}^{2}\right)-\frac{1}{8} \bar{r}^{4} \overline{v v}_{z z z}=\frac{1}{\mathrm{We}}\left(\frac{\bar{r}}{\left(1+\bar{r}_{z}^{2}\right)^{1 / 2}}+\frac{\bar{r}^{2} \bar{r}_{z z}}{\left(1+\bar{r}_{z}^{2}\right)^{3 / 2}}\right)_{z}+\frac{1}{\operatorname{Re}}\left(3 \bar{r}^{2} \bar{v}_{z}-\frac{1}{8}\left(\bar{r}^{4} \bar{v}_{z z}\right)_{z}\right)_{z}+\frac{\bar{r}^{2}}{\mathrm{Fr}}
\end{gathered}
$$

The numerical scheme employs a staggered formulation. An upwind scheme is used to approximate all convective terms, and a second-order central differencing scheme is employed to represent all other operators. Inflow conditions are enforced using Dirichlet conditions, and convective outflow conditions are used at the exit of the computational domain. The discretized equations are solved implicitly using an iterative scheme.

\section{B. Solution of perturbed flow}

To study hydrodynamic instabilities of a weakly non-parallel flow, a multiscale analysis is used [18]. A slow varying coordinate variable, $X$, is introduced as

$$
X=\varepsilon z
$$

in which $\varepsilon$ is defined as

$$
\varepsilon=\frac{L_{\text {inst }}}{L_{\text {mean }}},
$$

and measures the non-parallel effect of mean flow [18]. $L_{\text {mean }}$ is the characteristic streamwise inhomogeneities of the mean flow and $L_{\text {inst }}$ is the characteristic length scale of the instability (See Fig. 1). $L_{\text {inst }}$ is assumed to be the wavelength of the perturbation wave and $L_{\text {mean }}$ is defined as

$$
L_{\text {mean }}=\frac{\bar{v}}{d \bar{v} / d z} .
$$

To assess the validity of our perturbation analysis, we evaluate $\varepsilon$ from Eq. (8) as function of operating parameters. Results of this analysis for a fixed Reynolds number of 100 are illustrated in Fig. 2. It can be seen that for high Weber numbers where jet velocity is high, the effect of gravity is not important while for small Weber numbers, gravity could be effective. This figure shows that $\varepsilon$ remains less than unity for $B o<1$ and therefore justifying the weakly non-parallel mean flow approximation. 


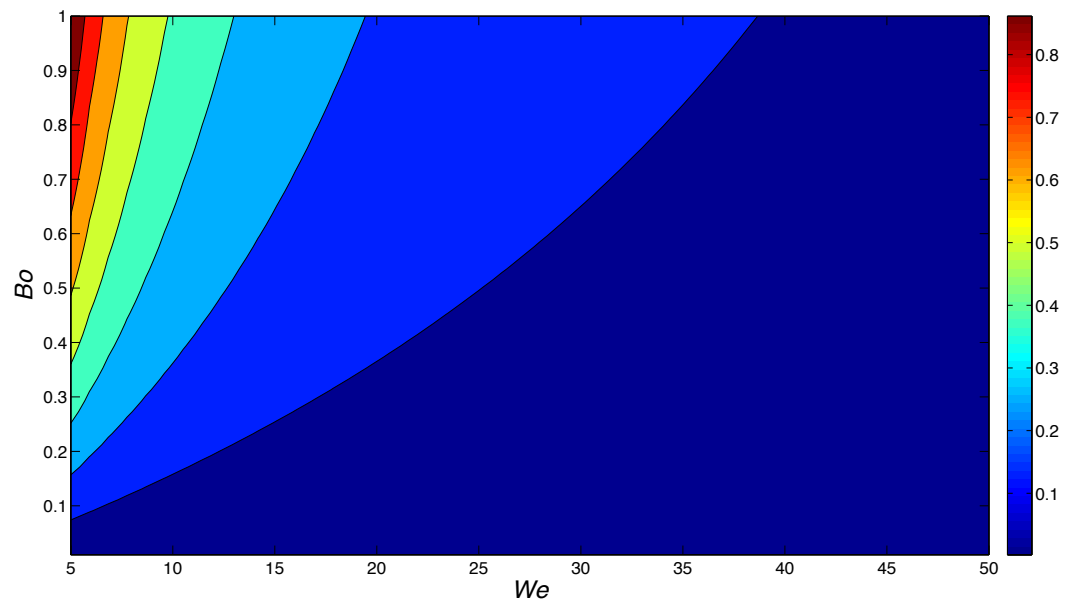

Fig. 2. Variation of non-parallel flow indicator, $\varepsilon$, with $W e$ and $B o$ numbers at $R e=100$

Toward a spatial analysis of Eqs. (1) and (2), the perturbed radius and perturbed velocity are represented by normal modes of the form,

$$
\begin{aligned}
& r^{\prime}=\left[\hat{r}_{0}(X)+\varepsilon \hat{r}_{1}(X)\right] \exp \left[i\left(\frac{1}{\varepsilon} \int^{X} \alpha(X) d X-\omega t\right)\right], \\
& v^{\prime}=\left[\hat{v}_{0}(X)+\varepsilon \hat{v}_{1}(X)\right] \exp \left[i\left(\frac{1}{\varepsilon} \int^{X} \alpha(X) d X-\omega t\right)\right],
\end{aligned}
$$

where $\hat{r}_{0}(X), \hat{r}_{1}(X)$ and $\hat{v}_{0}(X), \hat{v}_{1}(X)$ are amplitude components of disturbances of jet radius and velocity, respectively. Additionally, since we are considering a spatial instability analysis $\omega$ is the real-valued frequency, and $\alpha=\alpha_{r}+i \alpha_{i}$ is the complex-valued, whose real and imaginary parts represent the wavenumber and the growth rate in the axial direction, respectively. Linearized expansion of Eqs. (1) and (2) by (10) and (11) yields the following set of equations,

$$
\left[\begin{array}{ll}
A & B \\
C & D
\end{array}\right]\left\{\begin{array}{ll}
\hat{r}_{0} & \hat{r}_{1} \\
\hat{v}_{0} & \hat{v}_{1}
\end{array}\right\}=\left[\begin{array}{cc}
0 & M \\
0 & N
\end{array}\right],
$$

where the matrix components are defined in the Appendix. Nontrivial solutions of the two homogeneous equations of (12), yield the following dispersion relation:

$$
D(\omega, \alpha, X)=A D-B C=0,
$$

or in polynomial form,

$$
\begin{aligned}
& \left(\frac{i \bar{r}^{2}}{8 R e}\right) \alpha^{5}-\left(\frac{1}{8}-\frac{\bar{r}^{3}}{2 W e}+\frac{i \bar{r}^{4} \omega}{8 R e}\right) \alpha^{4}+\left(\frac{\bar{r}^{2} \omega}{4}+\frac{3 i}{\operatorname{Re}}\right) \alpha^{3}- \\
& \left(\frac{1}{\bar{r}^{2}}+\frac{\bar{r}^{4} \omega^{2}}{8}+\frac{\bar{r}}{2 W e}+\frac{3 i \bar{r}^{2} \omega}{\operatorname{Re}}\right) \alpha^{2}+\left(2 \omega+\frac{i \bar{r}^{2}}{F r}\right) \alpha-\left(\bar{r}^{2} \omega^{2}\right)=0 .
\end{aligned}
$$

The amplitude of the perturbations is found by solving the non-homogeneous equations in (12). After simplification, the following ordinary differential equation is obtained,

$$
P(X) \frac{d \hat{r}_{0}(X)}{d X}+Q(X) \hat{r}_{0}(X)=0,
$$

where $P(X)$ and $Q(X)$ are defined in the Appendix. Solution of this ordinary differential equation can be written as

$$
\hat{r}_{0}(X)=\hat{r}_{0}(0) \exp \left(\int^{X}-\frac{Q(X)}{P(X)} d X\right) .
$$




\section{Results}

For the case of zero Bond number, dispersion curves as a function of frequency for three different Reynolds numbers are presented in Fig. 3. The maximum growth rate and the corresponding wavenumber characterize the fastest growing (or the most probable) waves on the liquid surface that are eventually responsible for the breakup. From this figure, it can be seen that viscosity dampens the growth rate and shifts the maximum growth toward lower frequencies, resulting in the formation of larger droplets. Fig. 3 shows that in the case of zero gravity, the growth rate profiles are in excellent agreement with classical results [5], serving as verification for the developed stability formulation based on Cosserat's equations.

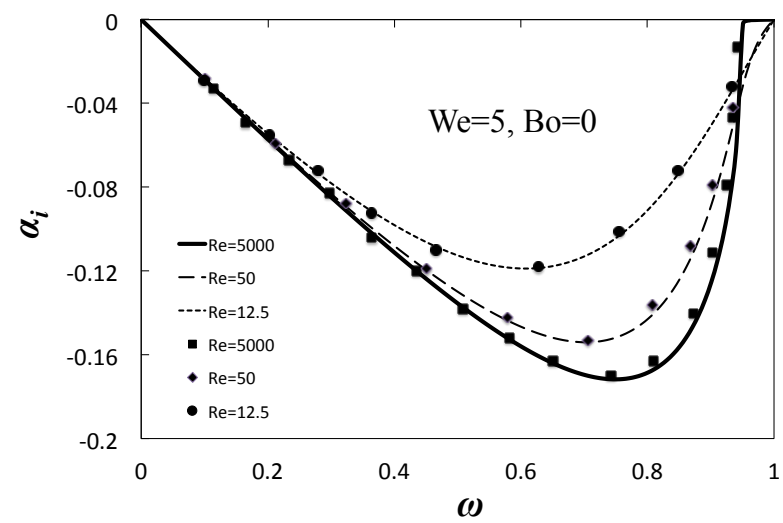

Fig. 3. Comparison of the growth rate obtained from present model (lines) and results of Leib and Goldstein [5] (symbols).

The jet profile for a series of representative $\mathrm{We}$ and $\mathrm{Fr}$ numbers are plotted in Fig. 4. The mean flow profile is calculated from numerical solution of equations (5) and (6) as described in part A. The fluctuating jet radius is calculated from Eqs. (10) and (14) assuming initial amplitude of $\hat{r}_{0}(0)=0.01$. Results confirm that for low Weber numbers and high Bond numbers, the mean flow is not parallel and a nonparallel analysis is required to perform a stability analysis.
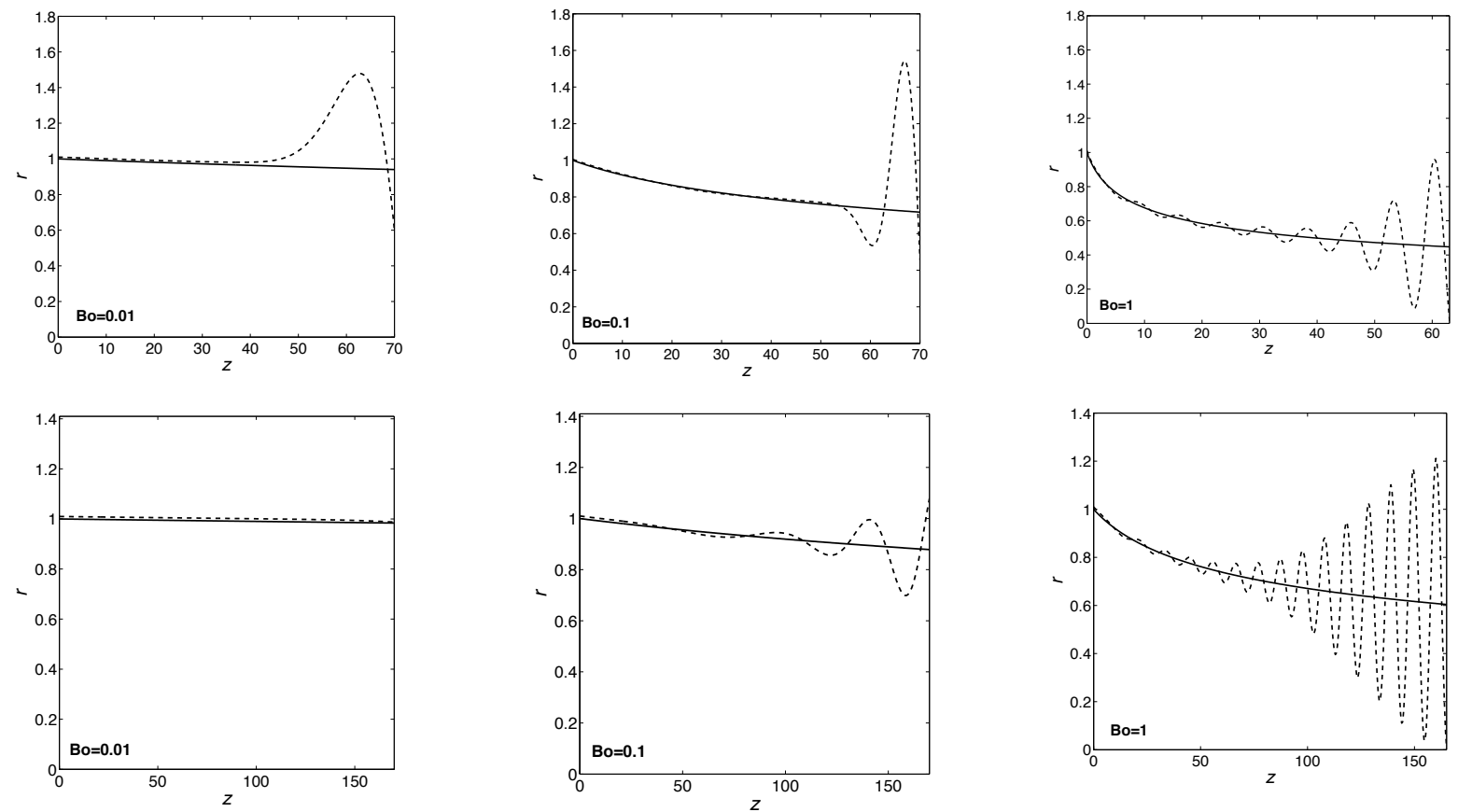

Fig. 4. Variation of jet radius (solid line: base flow, dashed line: perturbed flow) with axial distance for various Bond numbers at $W e=5$ (top) and $W e=50$ (bottom). 
Figures 5 and 6 show the effect of gravity on the amplification of disturbances for the selected cases of $\mathrm{We}=5,50$. From the results it can be seen that by increasing the Weber number, the instability growth rate is decreased which is in agreement with the well-known breakup curves [19], describing the shortening of the breakup length by reducing the jet velocity. Importantly, the results show that by increasing the Bond number, the instability grows faster. In addition, the range of unstable wavenumbers increases and the peak of the curve shifts towards higher frequencies. These results are in agreement with experiments [20,21], showing that the breakup length is reduced under consideration of gravity, resulting in the formation of smaller droplets.

$B o=0.01$
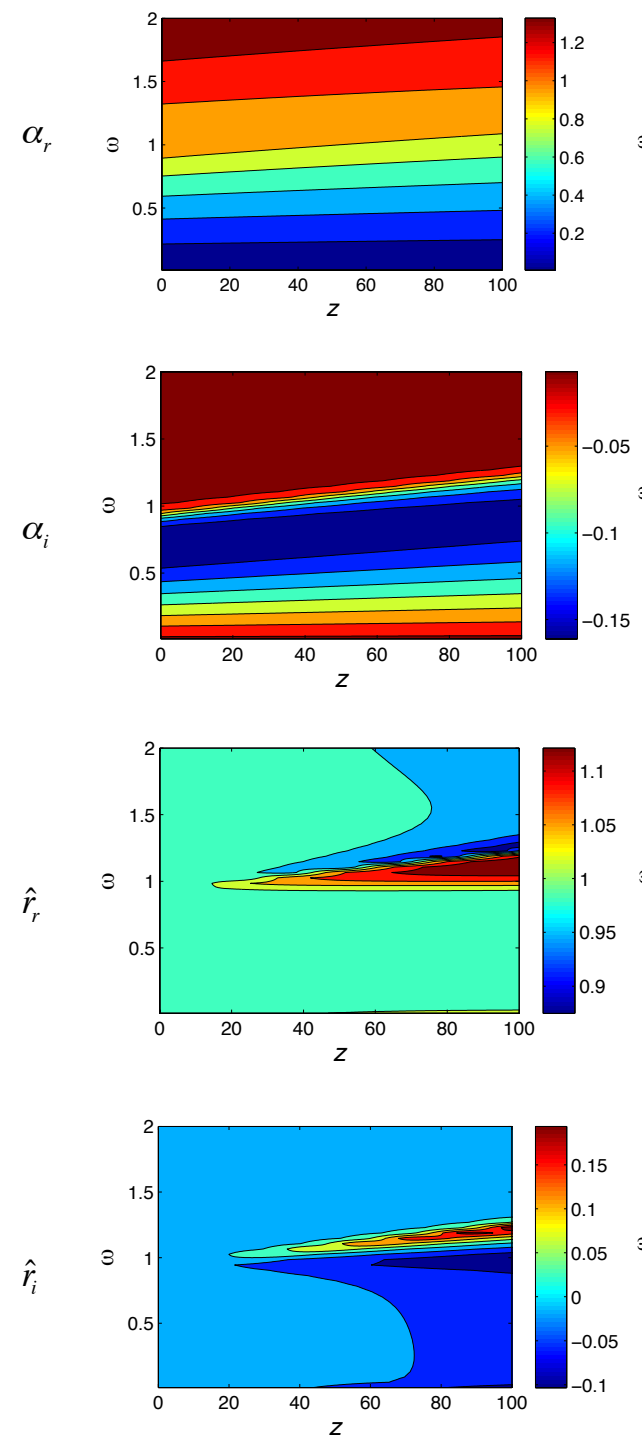

$B o=0.1$
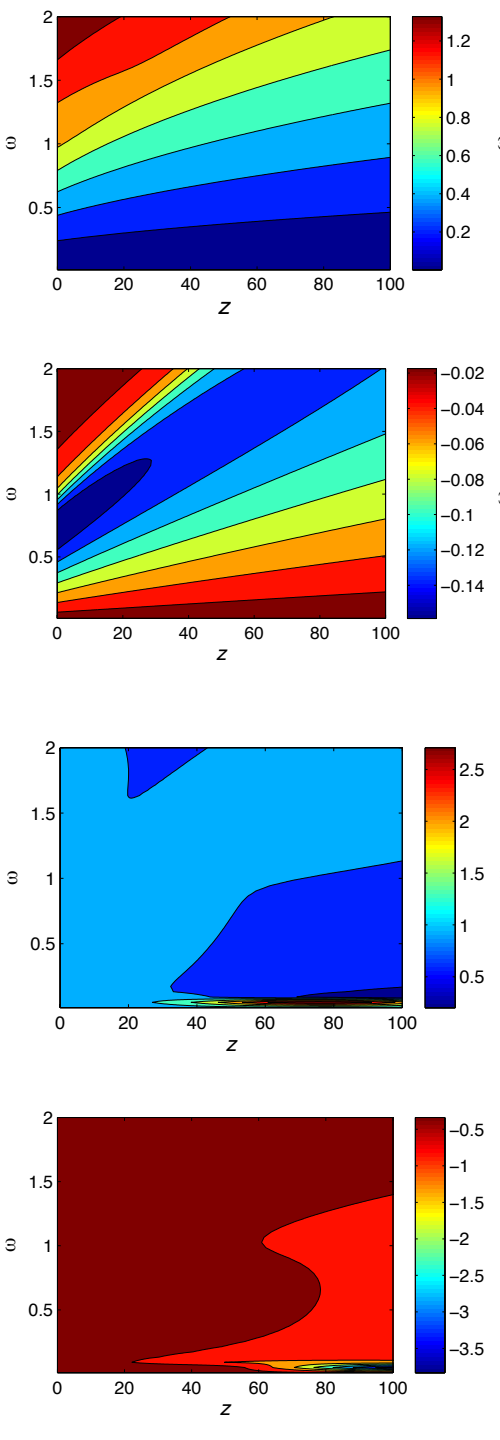

$B o=1$
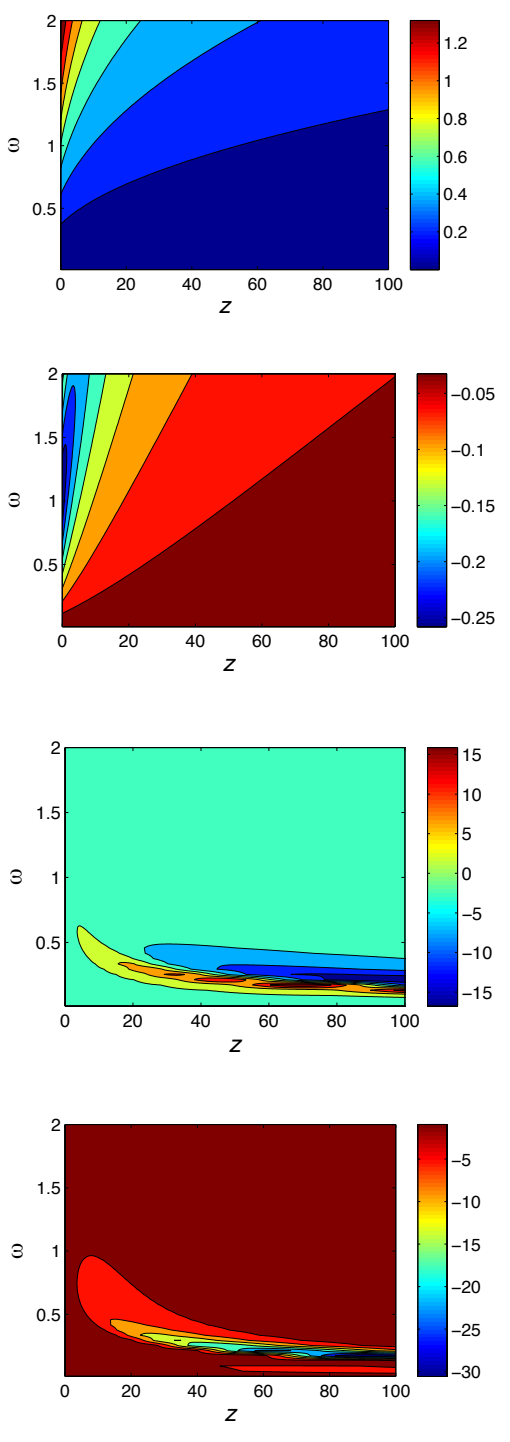

Fig. 5. Wavelength, $\alpha_{r}$, growth rate, $\alpha_{i}$, and real, $\hat{r}_{r}$, and imaginary, $\hat{r}_{i}$ parts of disturbance amplitude for different Bond numbers $(W e=5, R e=100)$. 

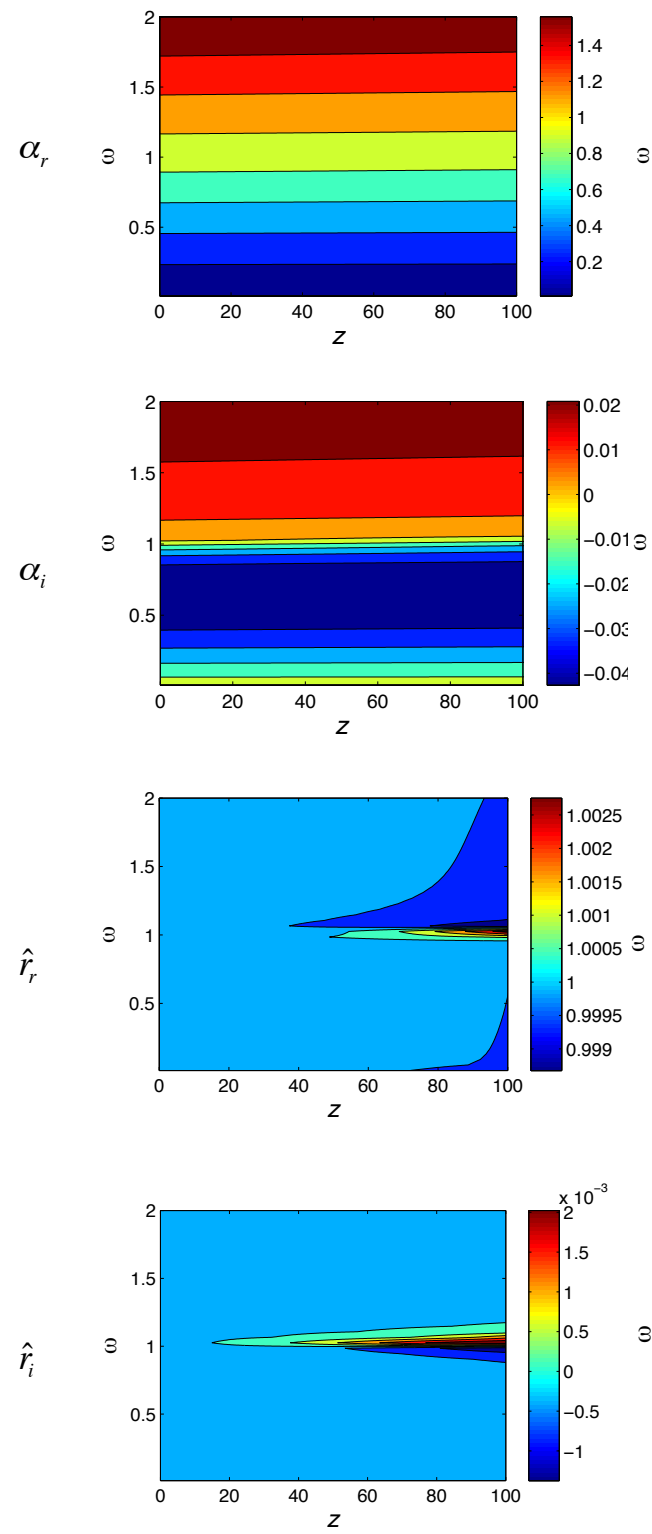

$B o=0.1$
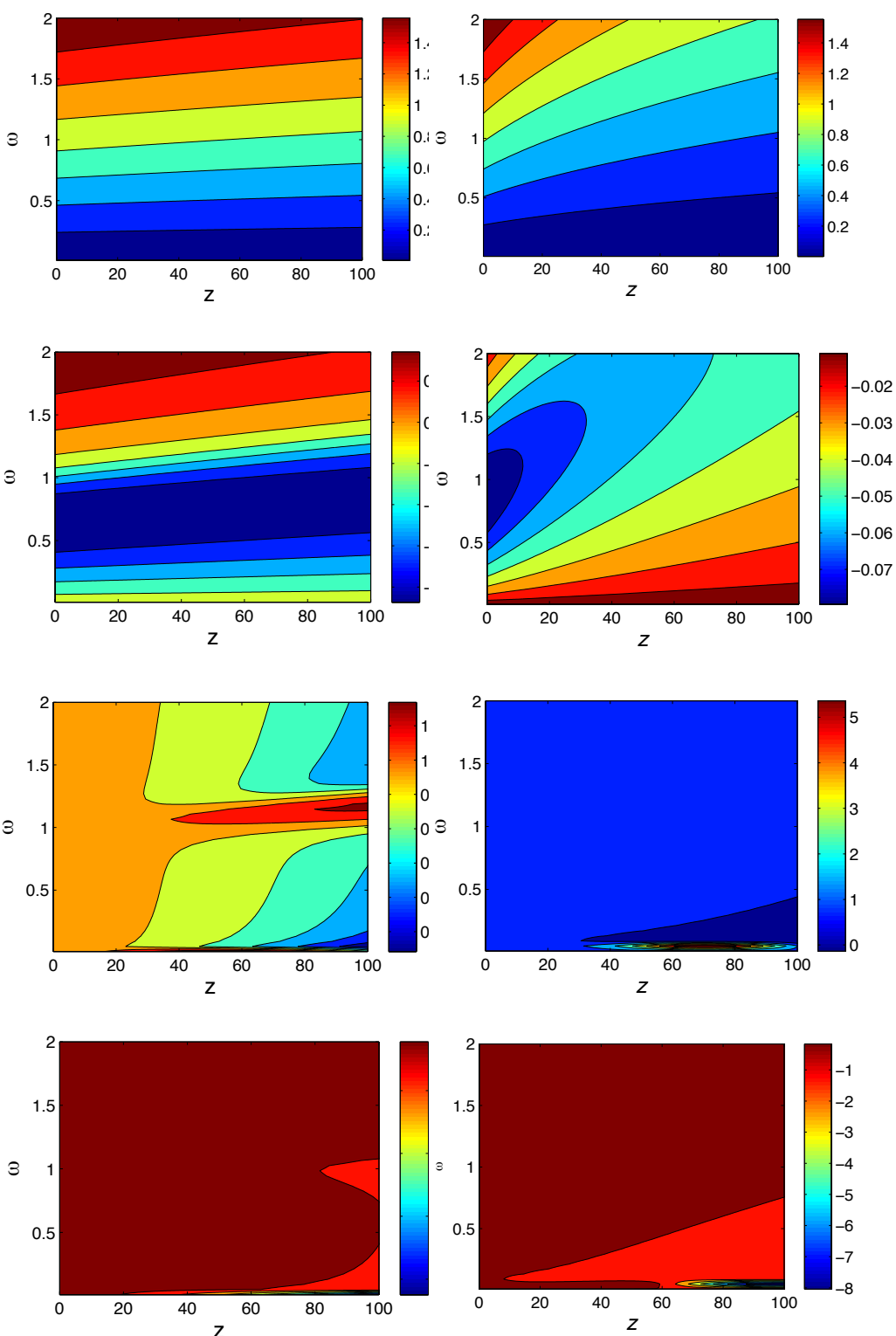

Fig. 6. Wavelength, $\alpha_{r}$, growth rate, $\alpha_{i}$, and real, $\hat{r}_{r}$, and imaginary, $\hat{r}_{i}$ parts of disturbance amplitude for different Bond numbers $(W e=50, R e=100)$.

The liquid jet breaks up in a streamwise location where the magnitude of the fluctuating part, as shown in Eq. (4), becomes equal to the mean jet radius. The effect of gravity on the jet breakup length is shown in Fig. 7. The results show that by increasing the Bond number, the breakup length is decreased, confirming the experiments by Howes and coworkers $[20,21]$.

The critical Weber number, below which a liquid jet (without gravity effects) is absolutely unstable, and above which the jet is convectively unstable, was found by Leib \& Goldstein [4] using the criteria of Briggs [22]. The critical Weber number for the limit $\mathrm{Re} \rightarrow \infty$ was evaluated from Eq. (14) using the same saddle-point criterion [22], resulting in a value of 3.10, which is very close to the value of $\pi$ found by Leib and Goldstein [4]. The dependence of the critical Weber number as function of Reynolds number and different Froude-numbers is depicted in Fig. 8. For the viscous case, the current analysis shows that for higher Froude numbers, where gravity effects are negligible, 
the critical Weber number approaches the asymptotic value of Rayleigh-Chandrasekhar calculated by Leib and Goldstein [5]. As can be seen, the critical Weber number decreases with decreasing Froude numbers.

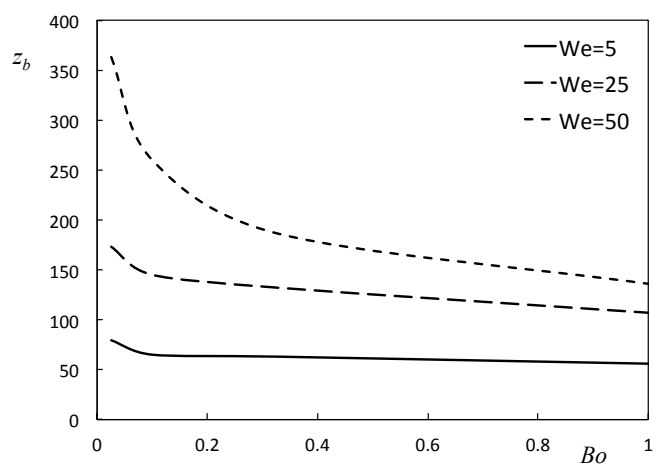

Fig. 7. Breakup length, $z_{b}$ as function of Weber number for various Bond numbers for the most unstable frequencies.

$$
\left(\boldsymbol{R} \boldsymbol{e}=\mathbf{1 0 0}, \hat{r}_{0}(0)=0.01\right)
$$

Surface tension is the source of instabilities in low-speed jets. If the capillary force is sufficiently larger than the inertia, the disturbances propagate both upstream and downstream, whereas in the opposite event, the inertia is dominant over the surface tension force and the disturbances can only propagate in the downstream direction. In a downward-pointing jet, gravity enhances the inertia and increases the convective effects, therefore disturbances are not able to propagate upstream except for low Weber numbers. Part of the disturbances will propagate back to the nozzle tip to prevent the formation of a jet of any length. Dripping is a candidate for the manifestation of absolute instability and the transition between absolute and convective instability is comparable with the transition from dripping to jetting [19]. Results in Fig. 8 are in agreement with those of Clanet and Lasheras [23] and show that by decreasing the Froude number, the transition from dripping to jetting occurs at lower Weber numbers.

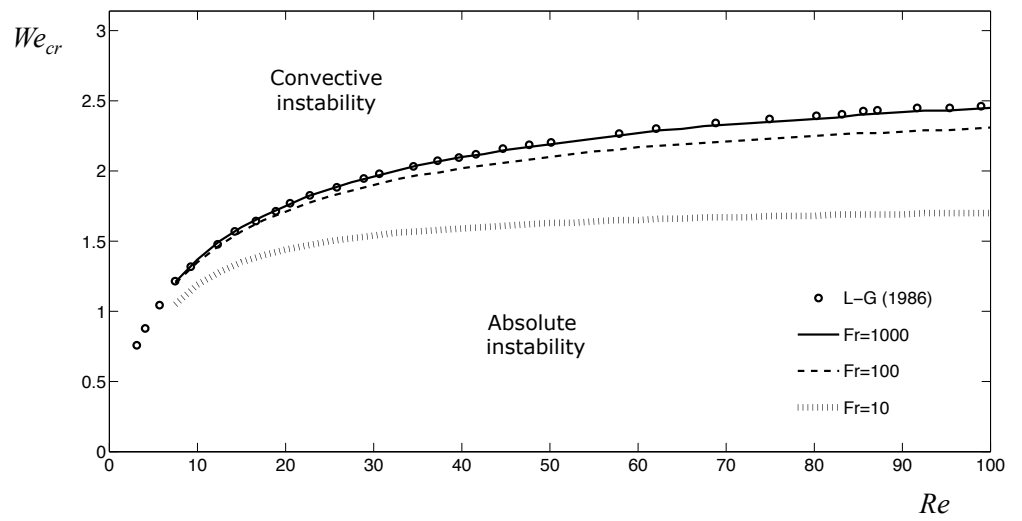

Fig. 8. Critical Weber numbers versus Reynolds number for various Froude numbers.

\section{Conclusion}

The linearized form of the one-dimensional Cosserat's equations has been used to study the wave propagation in a liquid jet under consideration of gravitational effects. Using multiscale analysis, a closed form dispersion equation has been derived which considers effects of gravity. Through a systematic analysis, the variation in disturbance growth rate was investigated for a specified range of Bond numbers. For the special case of zero gravity, the presented formulation is in agreement with classical results of Leib and Goldstein [4,5]. The results presented in this work reveal that by increasing the gravity, the jet instability increases, maximum growth rate shifts toward shorter waves, and the cut-off frequency is increased. Further reducing the Froude number, decreases the area of absolute instability. 


\section{Appendix}

$$
\begin{aligned}
& A=-i \omega+i \bar{v} \alpha \\
& B=\frac{i \bar{r} \alpha}{2} \\
& C=\frac{1}{W e}\left(i \bar{r}^{2} \alpha^{3}-i \alpha\right)-\frac{2 \bar{r}}{F r} \\
& D=-i \bar{r}^{2} \omega+i \alpha-\frac{i}{8} \bar{r}^{4} \alpha^{2} \omega+\frac{1}{8} i \bar{r}^{2} \alpha^{3}+\frac{1}{\operatorname{Re}}\left(\frac{1}{8} \bar{r}^{4} \alpha^{4}+3 \bar{r}^{2} \alpha^{2}\right) \\
& M=M_{1} \hat{r}_{0}+M_{2} \hat{r}_{0 X}+M_{3} \hat{v}_{0}+M_{4} \hat{v}_{0 X} \\
& N=N_{1} \hat{r}_{0}+N_{2} \hat{r}_{0 X}+N_{3} \hat{v}_{0}+N_{4} \hat{v}_{0 X} \\
& M_{1}=-\frac{\bar{v}_{X}}{2} \\
& M_{2}=-\bar{v} \\
& M_{3}=-\bar{r}_{X} \\
& M_{4}=-\frac{\bar{r}}{2} \\
& N_{1}=-2 \overline{r v v_{X}}-\frac{1}{W e}\left(\overline{r r_{X}} \alpha^{2}+3 \bar{r}^{2} \alpha \alpha_{X}\right)+\frac{6}{\operatorname{Re}}\left(i \bar{r} \bar{v}_{X} \alpha\right) \\
& N_{2}=\frac{1}{W e}\left(1-3 \bar{r}^{2} \alpha\right)^{2} \\
& N_{3}=-\bar{r}^{2} \bar{v}_{X}+\frac{1}{2} \bar{r}^{3} \bar{r}_{X} \omega \alpha-\frac{1}{2} \overline{r r}_{X} \alpha^{2}+\frac{1}{8} \bar{r}^{4} \omega \alpha_{X}-\frac{3}{8} \bar{r}^{2} \alpha \alpha_{X}+\frac{i}{\operatorname{Re}}\left(\bar{r}^{3} \bar{r}_{X} \alpha^{3}+\frac{3}{4} \bar{r}^{4} \alpha \alpha_{X}+6 \overline{r r_{X}} \alpha+3 \bar{r}^{2} \alpha_{X}\right) \\
& N_{4}=-1+\frac{1}{4} \bar{r}^{4} \alpha \omega-\frac{3}{8} \bar{r}^{2} \alpha^{2}+\frac{i}{\operatorname{Re}}\left(\frac{1}{2} \bar{r}^{4} \alpha^{3}+6 \bar{r}^{2} \alpha\right) \\
& P=\left(N_{2}-\frac{C}{A} M_{2}\right)-\left(N_{4}-\frac{C}{A} M_{4}\right) \frac{A}{B} \\
& Q=\left(N_{1}-\frac{C}{A} M_{1}\right)-\left(N_{3}-\frac{C}{A} M_{3}\right) \frac{A}{B}-\left(N_{4}-\frac{C}{A} M_{4}\right)\left(\frac{A_{X} B-A B_{X}}{B^{2}}\right)
\end{aligned}
$$




\section{Acknowledgment}

Financial support through the Office of Naval Research and National Science Foundation is gratefully acknowledged.

\section{References}

[1] Eggers, J. and Villermaux E.,” Physics of liquid jets” Rep. Prog. Phys., Vol. 71, 036601, 2008, 79 pp.

[2] Rayleigh," On the capillary phenomena of jets" Proc. R. Soc., Vol. 29, 1879, pp. 71-97.

[3] Keller, J.B., Rubinow, S.I., and Tu Y.O.," Spatial instability of a jet” Phys. Fluids, Vol. 16, 1973, pp. $2052-2055$.

[4] Leib S.J. and Goldstein, M.E.," The generation of capillary instability on a liquid jet" J. Fluid Mech., Vol. 168, 1986, pp. 479-500.

[5] Leib S.J. and Goldstein, M.E.," Convective and absolute instability of a viscous liquid jet” Phys. Fluids, Vol. 29, 1986, pp. 952-954.

[6] Ambravaneswaran B., Subramani H.J., Phillips S.D., and Basaran O.A.," Dripping-jetting transition in a dripping faucet” Phys Rev Lett., Vol. 93, 034501, 2004, 4 pp.

[7] Montanero, J.M., Herrada, M.A., Ferrera, C., Vega, E.J., and Ganan-Calvo, A.M.," On the validity of a universal solution for viscous capillary jets" Phys. Fluids, Vol. 23, 122103, 2011, 12 pp.

[8] Green A.E., "On the non-linear behavior of fluid jets". Int. J. Eng. Sci., Vol. 14, 1976, pp. 49-63.

[9] Garcia F.J. and Castellanos, A., "One-dimensional models for slender axisymmetric viscous liquid jets " Phys. Fluids, Vol. 6, 1994, pp. 2676-2689.

[10] Eggers, J., "Nonlinear dynamics and breakup of free-surface flows" Rev. Mod. Phys., Vol. 69, 1997, pp. 865-929.

[11] Bogy, D.B.," Use of one-dimensional Cosserat theory to study instability in a viscous liquid" Phys. Fluids, Vol. 21, 1978, pp. 190-197.

[12] Bogy, D.B.," Wave propagation and instability in a circular semi-infinite liquid jet harmonically forced at the nozzle" $J$. Appl. Mech., Vol. 45, 1978, pp. 469-474.

[13] Bogy, D.B., "Break-up of a liquid jet: second perturbation solution for one-dimensional Cosserat theory" IBM J. Res. Dev., Vol. 23, 1979, pp. 87-92.

[14] Bogy, D.B.," Break-up of a liquid jet: third perturbation Cosserat solution” Phys. Fluids, Vol. 22, 1979, pp. $224-230$.

[15] Amini, G. and Dolatabadi, A., "Capillary instability of elliptic liquid jets" Phys. Fluids, Vol. 23, No. 8, 084109, 2011,9 pp.

[16] Amini, G. and Dolatabadi, A., "Axis-switching and breakup of elliptic liquid jets" Int. J. Multip. Flow, Vol. 42, 2012, pp. 96-

[17] Bogy, D.B.," Drop formation in a circular liquid jet” Ann. Rev. Fluid. Mech., Vol. 11, 1979, pp. 207-228.

[18] Huerre, P. and Rossi, M., "Hydrodynamics and nonlinear instabilities" Ed. by Godreche, C., and Manneville, P., Camb. Univ. Press, 1998, Chap. 2.

[19] Lin, S.P., and Reitz, R.D., "Drop and spray formation from a liquid jet” Ann. Rev. Fluid Mech., Vol. 30, 1998, pp. 85-105.

[20] Cheong, B.S., and Howes, T.," Capillary jet instability under the influence of gravity" Chem. Eng. Sci., Vol. 59, 2004, pp. 2145-2157.

[21] Edwards, A.P.R., Osborne, B.P., Stoltzfus, J.M., Howes, T., and Steinberg, T.A., "Instabilities and drop formation in cylindrical liquid jets in reduced gravity" Phys. Fluids, Vol. 14, 2002, pp. 3432-3438

[22] Briggs, R.J., "Electron-Stream Interaction with Plasmas" MIT Press, Cambridge, MA, 1964.

[23] Clanet, C. and Lasheras, J.C.," Transition from dripping to jetting” J. Fluid Mech., Vol. 383, 1999, pp. 307-326. 